\title{
THEMATISCHER SCHWERPUNKT 2018: Revolutionen
}

Auf das Jahr 2018 fallen zwei Revolutionsjubiläen: der 170. Jahrestag der europäischen Revolution von 1848/49 und der 100. Jahrestag der deutschen Revolution vom November 1918. Auch die Studentenbewegung von 1968 jährt sich zum 50. Mal. Diese Tatsache nimmt ConviviUm zum Anlass, um zu Reflexionen über das Thema „Revolution“ aus germanistischer Perspektive einzuladen.

Revolutionen sind zumeist Thema geschichtswissenschaftlicher Studien, die neue Erkenntnisse zu Ursachen, Verlauf und Wirkungen, zum ideengeschichtlichen Kontext oder zur Bedeutung politischer Revolutionen vorstellen. Das Interesse der Germanistik richtet sich hingegen auf das Verhältnis einzelner Schriftstellerinnen und Schriftsteller zur Revolution und auf die Thematisierung politischer Umwälzungen in literarischen Werken. Auch die Rolle von Literatur und Sprache in Zeiten revolutionärer Aufbrüche und die Auswirkungen von Revolutionen auf Veränderungen in der Literatur (Programmatiken, Schreibweisen) bilden einen Untersuchungsgegenstand. Im Zentrum der Forschung stehen dabei das Jahrhundert der Revolutionen von der Französischen Revolution bis zu den europäischen Revolutionen des Jahres 1848/49, die Revolutionen in und um den Ersten Weltkrieg sowie revolutionäre Bewegungen nach 1945 mit dem Schwerpunkt des Jahres 1989. Diese Perspektive lässt sich durch komparatistische Studien, etwa zur deutschen und polnischen, aber auch $\mathrm{zu}$ anderen Literaturen erweitern. So kann in den einzelnen Beiträgen den kulturell bedingten Unterschieden in den literarischen Bildern etwa der Französischen Revolution oder der Umbrüche des Jahres 1989 ebenso nachgegangen werden wie der Position, die Schriftstellerinnen und Schriftsteller sowie literarische Institutionen in den Revolutionen des 19. und 20. Jahrhunderts in verschiedenen Ländern einnahmen. Welche Auswirkungen hatten revolutionäre Veränderungen auf die Literatur? Wie kündigten literarische Werke gesellschaftliche Umbrüche an? Unter einem weiten Begriff von „Revolution“ können auch mentalitäts- und ideengeschichtliche, religiöse, ökonomische und technische Umbrüche sowie die sexuelle und ökologische Revolution des 20. Jahrhunderts verstanden werden. Nicht zuletzt sind Beiträge von Interesse, in denen literarische Seismographien der sich gegenwärtig ankündigenden Veränderungen thematisiert werden. 
Der Schwerpunkt umfasst nicht nur Untersuchungen zum Verhältnis von Literatur und Sprache auf der einen, Revolutionen und Mentalitätswechseln auf der anderen Seite. Gegenstand der Reflexion können darüber hinaus Literaturrevolutionen sowie Paradigmenwechsel in der Geschichte der Germanistik sein. Interessant wäre es, die wissenschaftlichen Erträge einzelner ,turns', wie das ,turning' der Wissenschaft überhaupt, einer kritischen Analyse zu unterziehen. ,Revolutionen' in der Wissenschaft im Sinne des Kuhnschen Konzepts des Paradigmenwechsels führen zur Entwicklung neuer Theorien und legen den Fokus auf neue Forschungsfragen, die mit neuen Methoden untersucht werden. In der Geschichte der Sprachwissenschaft werden mehrere Wendepunkte als ,revolutionär" bezeichnet, u. a. die junggrammatische Schule und der Paradigmenwechsel durch die Entwicklung des Strukturalismus. Die Publikation von Ferdinand de Saussures Cours de linguistique générale (1916) bildete nur den Anfang für die Entwicklung verschiedener strukturalistischer Schulen. Mit Noam Chomskys Syntactic Structures (1957) begann durch die Generative Grammatik eine neue ,Revolutionsperiode‘. Der Aufschwung der Informationstechnologie führt heute zur digitalen Revolution. Insofern wäre auch die Frage von Interesse, welche Zukunftschancen die Digital Humanities haben.

\section{Literatur}

ARENDT, HANNAH (1959): Die ungarische Revolution und der totalitäre Imperialismus. München.

ARENDT, HANNAH (1974): Über die Revolution. München.

BARFoOt, CEDRIC C. / D'HAEN, THEO (eds.) (1991): Tropes of revolution. Writer's reactions to real and imagined revolutions, 1789-1989 (1991). Amsterdam / Atlanta.

BIEBER, HANS JOACHIM (1991): Bürgertum in der Revolution. Hamburg.

BLOCK, RUSSEL LLOYD (1986): Revolution und Revision in der generativen Theoriebildung. Tübingen.

BRANDT, MARION (2002): Für eure und unsere Freiheit? Der Polnische Oktober und die Solidarność-Revolution in der Wahrnehmung von Schriftstellern aus der DDR. Berlin.

ChoŁuj, Bożena (1991): Deutsche Schriftsteller im Banne der Novemberrevolution 1918. Bernhard Kellermann, Lion Feuchtwanger, Ernst Toller, Erich Mühsam, Franz Jung. Wiesbaden.

EtZler, Melissa / Layne, Priscilla (eds.) (2010): Rebellion and revolution. Defiance in German language, history and art. Newcastle upon Tyne.

GRIEWANK, KARL (1992): Der neuzeitliche Revolutionsbegriff. Entstehung und Entwicklung. Frankfurt a. M. 
HARTH Dietrich / ASSMAnn, JAN (eds.) (1992); Revolution und Mythos. Frankfurt a. M. Hoyningen-Huene, Paul (1989): Die Wissenschaftsphilosophie Thomas S. Kuhns. Rekonstruktion und Grundlagenprobleme. Wiesbaden.

KING, IAN und ILLE, STEFFEN (eds.) (2015): Schriftsteller und Revolution. Dokumentation der Jubiläumstagung 2013. St. Ingbert.

KitTstein, Ulrich / Zeller, Regine (eds.) (2009): „,Friede, Freiheit, Brot! “ Romane zur deutschen Novemberrevolution (2009). Amsterdamer Beiträge zur neueren Germanistik 71.

Kluge, Ulrich (1985): Die deutsche Revolution 1918/19. Staat, Politik und Gesellschaft zwischen Weltkrieg und Kapp-Putsch. Frankfurt a. M.

KoENEN, GERD (2002): Das rote Jahrzehnt. Unsere kleine deutsche Kulturrevolution. Frankfurt a. M.

Kunn, Thomas S. (2001): Die Struktur wissenschaftlicher Revolutionen. Frankfurt a. M. [Kunn, Thomas S. (1962): The Structure of Scientific Revolutions. Chicago].

LANDAUER, Gustav (1977): Revolution. Einleitung Harry Pross. Nachwort Erich Mühsam. Berlin.

RiCHTER, Michael ( $\left.{ }^{3} 1995\right):$ Die Revolution in Deutschland 1989/90. Anmerkungen zum Charakter der ,,Wende“. Dresden.

SANGMEISTER, DIRK / MUlsow, MARTIN (eds.) (2014): Subversive Literatur. Erfurter Autoren und Verlage im Zeitalter der Französischen Revolution (1780-1806) (2014). Göttingen.

SCHINGS, HANS-JÜRGEN (2012): Revolutionsetüden. Schiller - Goethe - Kleist. Würzburg.

STRELler, SiEGFRIED in Zusammenarbeit mit NAMOWICZ, TAdEUSZ (eds.) (1989): Literatur zwischen Revolution und Restauration. Studien zu literarischen Wechselbeziehungen in Europa zwischen 1789 u. 1835 (1989). Berlin / Weimar.

WISSENSCHAFTLICHES ZENTRUM II DER GESAMTHOCHSCHULE KASSEL - UNIVERSITÄT IN ZUSAMMENARBEIT MIT DER GEORG FORSTER-GESELLSCHAFT E.V. UND DEM STAATSTHEATER KASSEL (eds.): 1789. Deutsche Erfahrungen mit einer fremden Revolution (1990). Kassel.

WOLFERT, JUTTA (2004): Theatertexte zwischen Medien und Revolution 1989-1996. Achternbusch, Berg, Braun, Bukowski, Chatten, Czeslik, von Düffel, Enders, Goetz, Kroetz, Marber, Mueller, Müller, Rathenow, Reinshagen, Schneider, Specht, Teschke, Wildenhain. Berlin.

WoŁkowicz AnNA (2007): Mystiker der Revolution. Studien zum utopischen Diskurs um die Jahrhundertwende. Gustav Landauer, Frederik van Eeden, Erich Gutkind, Florens Christian Rang, Georg Lukács, Ernst Bloch. Warszawa.

$M B / D R S$ 